\title{
Ultra-Sensitive Ultrasound
}

\author{
Maranda Q. Herner ${ }^{1}$, Anna Maw ${ }^{1}$ \\ 1. Internal Medicine, University of Colorado- Anschutz Medical Campus, Aurora, USA \\ Corresponding author: Maranda Q. Herner, maranda.herner@cuanschutz.edu
}

\begin{abstract}
Lung ultrasound (LUS) is a dynamic, real-time, non-invasive bedside tool that offers increased sensitivity over standard imaging modalities in identifying pulmonary edema. This case highlights acute post-operative hypoxia secondary to pulmonary edema that was initially missed by chest radiography (CXR) and chest computed tomography (CT). The edema was diagnosed first on same day by bedside LUS, later seen on next day follow-up CXR and resolved with diuresis. LUS has demonstrated superior accuracy compared to CXR, but scant evidence compares it to CT. This case presentation serves to increase awareness of LUS as a highly sensitive and easy-to-use diagnostic tool for hospital providers in the evaluation of acute hypoxia.
\end{abstract}

Categories: Internal Medicine, Pulmonology, Quality Improvement

Keywords: lung ultrasound, point-of-care-ultrasound, pulmonary edema, diagnostic accuracy

\section{Introduction}

A multicenter study recently identified pulmonary edema, atelectasis and pleural effusions as frequent causes of post-operative hypoxia requiring over 24-hours of supplemental oxygen [1]. Lung ultrasound (LUS) is known to be more accurate than other bedside tools in the detection of pulmonary edema including auscultation and chest radiography (CXR) [2]. Its accuracy compared to chest computed tomography (CT) has not been well studied. This report presents a case of acute hypoxia secondary to pulmonary edema found on bedside LUS that was initially missed by both CXR and chest CT.

\section{Case Presentation}

A 66-year-old, non-obese woman with no significant past medical history initially presented with right knee pain. Synovial fluid analysis was consistent with septic arthritis for which she received joint wash out under general anesthesia. Postoperatively, she developed transient chest pain, tachypnea and new hypoxia with $\mathrm{PaO} 2$ of $59 \mathrm{mmHg}$ on room air, requiring $5 \mathrm{~L} / \mathrm{min}$ of oxygen to maintain a saturation above $90 \%$. Her initial post-operative CXR was read as nonspecific with bibasilar and perihilar opacities concerning for atelectasis or aspiration (Figure 1).

\author{
Received 04/03/2020 \\ Review began 04/09/2020 \\ Review ended 04/11/2020 \\ Published 04/20/2020 \\ (c) Copyright 2020 \\ Herner et al. This is an open access article \\ distributed under the terms of the \\ Creative Commons Attribution License \\ CC-BY 4.0., which permits unrestricted \\ use, distribution, and reproduction in any \\ medium, provided the original author and \\ source are credited.
}




\section{Cureus}

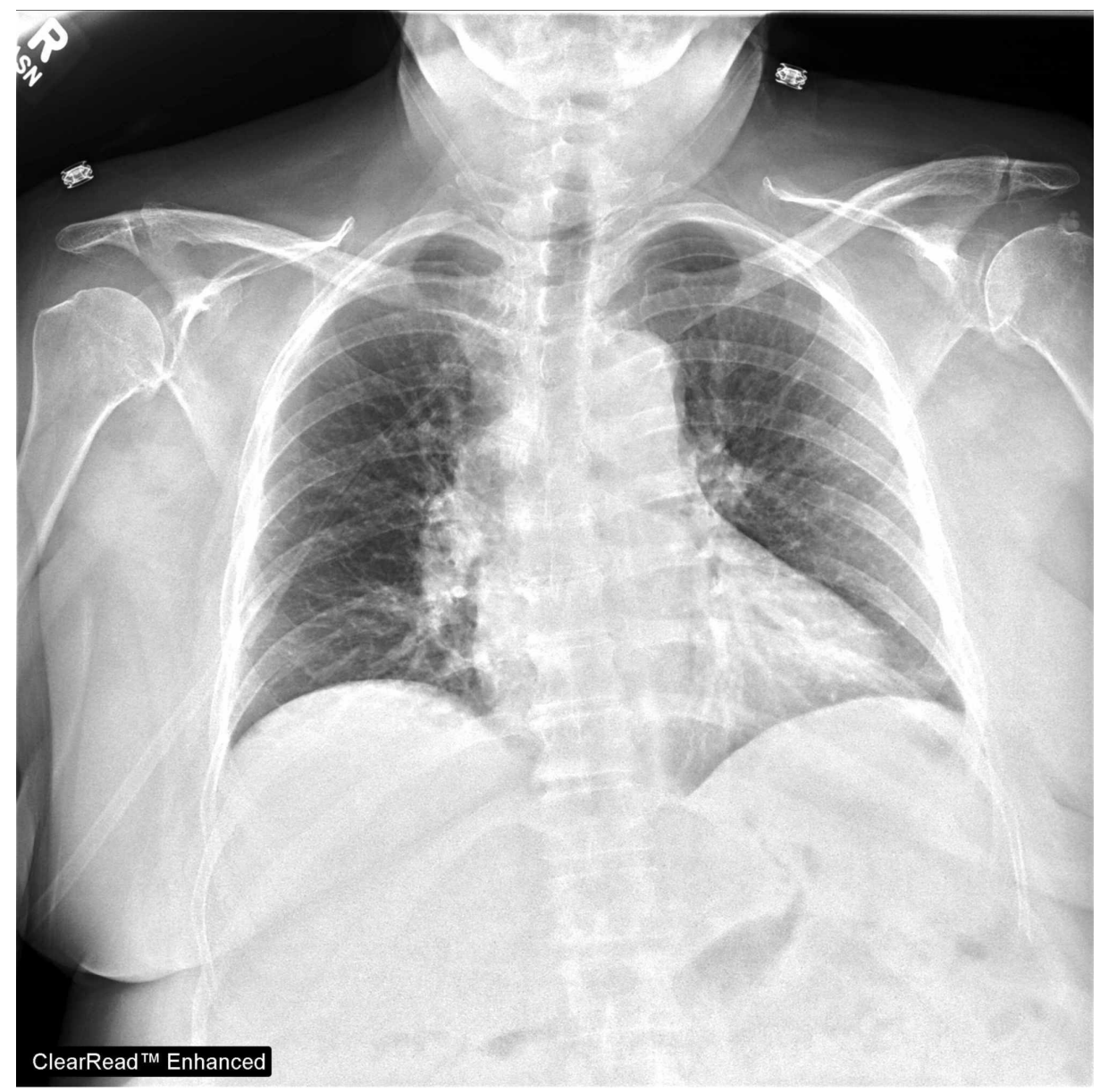

\section{FIGURE 1: Initial Chest Radiography (CXR), with no evidence of pulmonary edema}

The radiology report of the same day pulmonary embolism (PE) protocol chest CT was negative for PE, positive for bibasilar atelectasis and small bilateral effusions but without mention of pulmonary edema. Her troponin was negative, brain natriuretic peptide (BNP) was 253 , and arterial blood gas showed no hypercarbia and normal A-a gradient. Electrocardiogram was non-ischemic with sinus tachycardia. Echocardiogram showed right ventricular systolic pressure elevation to $41 \mathrm{mmHg}$ but no evidence of left ventricular systolic or diastolic dysfunction. Even after her chest pain resolved and her respiratory rate normalized, she remained hypoxemic.

Clinically, her 16-pound weight increase from admission, reported orthopnea, and mildly elevated brain natriuretic peptide (BNP) prompted a same day LUS which demonstrated multiple bilateral comet tails (Figure 2). 


\section{Cureus}

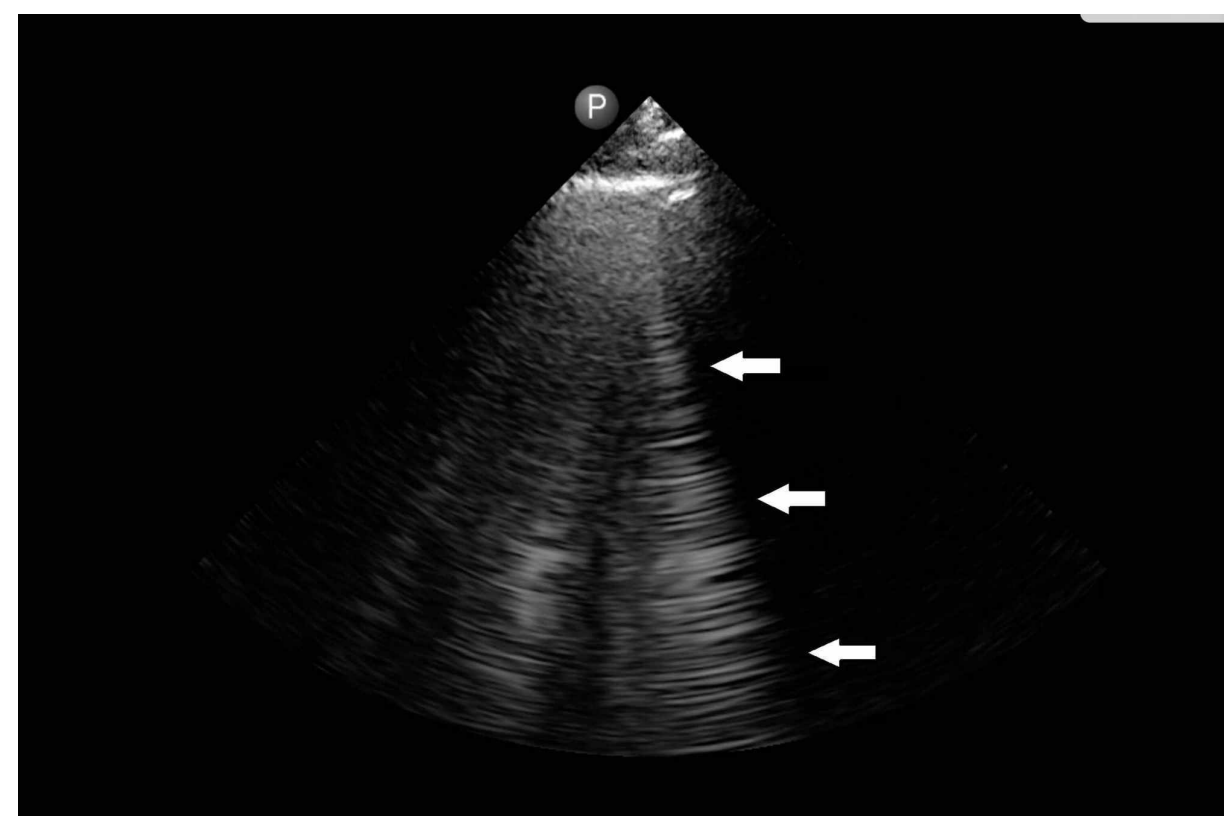

FIGURE 2: Lung Ultrasound, with multiple comet tails (also known as Blines)

One vertical comet tail (B-line) is indicated by the three white arrows.

While her oxygen requirements remained elevated and unchanged the following day, a repeat CXR noted enlarging bilateral effusions and evidence of vascular congestion consistent with pulmonary edema (Figure 3).

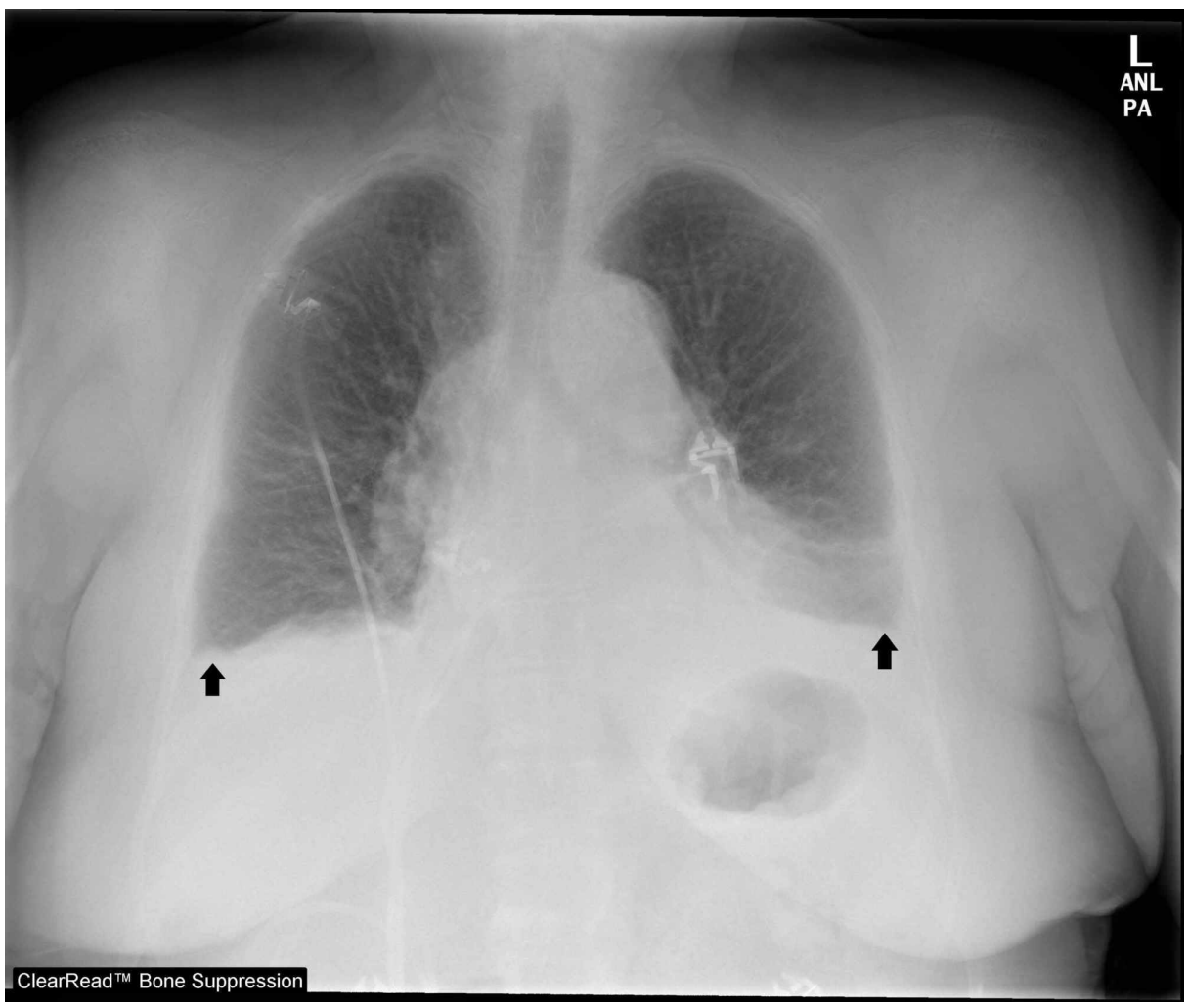

FIGURE 3: One Day Later, Repeat Chest Radiography (CXR)

Black arrows identify bilateral pleural effusions. CXR also notable for diffuse vascular congestion with pulmonary edema. 
The team suspected her hypoxemia resulted from a combination of pulmonary edema and hypoventilation related to atelectasis. Her mild pulmonary hypertension and mildly elevated BNP may reflect cardiogenic edema, though the echocardiogram was non-confirmatory for left ventricular dysfunction. Medication induced diuresis led to improvement on serial CXRs and resolution of her hypoxemia, consistent with pulmonary edema as the underlying etiology. At post-discharge follow up, she had returned to pre-admission weight and exhibited a $95 \%$ oxygen saturation on room air.

\section{Discussion}

As a portable, dynamic and minimally invasive tool, LUS can identify pulmonary edema in acute hypoxic respiratory failure (AHRF) through vertically oriented, hyperechoic lines of artifact termed comet tails or Blines, as seen in our patient (Figure 2). In an observational study, multiple B-lines on LUS demonstrated a sensitivity of $97 \%$ and specificity of $95 \%$ for pulmonary edema differentiated from other causes of AHRF [3].

CXR exhibits a false negative rate of $20 \%$ for detecting pulmonary edema and has been shown in a metaanalysis to be less sensitive than LUS [2]. Further, LUS provides a more accurate, real-time lung assessment as CXR has a known lag time in revealing both the initial presence and the resolution of pulmonary edema [4]. The dynamic nature of LUS has been well demonstrated in studies of patients receiving hemodialysis. LUS performed immediately before and after hemodialysis showed significant reduction in B-lines post dialysis, which correlated with weight reductions [5]. Studies monitoring response to diuretics have also noted LUS to demonstrate evidence of improvement sooner than CXR, highlighting LUS's earlier detection of changes in the dynamic process of pulmonary edema [6]. Consistent with these observations, this patient's first CXR failed to demonstrate the vascular congestion seen on the next day CXR. LUS offered this patient a more sensitive diagnostic tool for her early pulmonary edema.

While prior studies evaluating the accuracy of LUS have used chest CT as the reference standard, this case uniquely demonstrates failure of CT to diagnose acute pulmonary edema [2]. This raises the question of the sensitivity of LUS compared to CT which can be addressed in future studies.

\section{Conclusions}

LUS is a highly accurate, practical and dynamic bedside tool that should be considered in the evaluation of any patient with AHRF, particularly in early presentations such as in the post-operative patient. In this case, initial CXR and CT chest failed to capture evidence of pulmonary edema. However, the multiple comet tails on LUS and the patient's brisk response to diuretic therapy indicate that pulmonary edema was in fact present. This case supports the more frequent use of LUS as a highly accurate and evidence-based diagnostic tool for AHRF. Diagnoses can be accelerated, and subsequent treatment interventions applied sooner when LUS, being a new, more sensitive tool for pulmonary edema, is utilized early. The incorporation of LUS in routine hospital practices can significantly improve diagnostic accuracy and therefore patient care.

\section{Additional Information}

\section{Disclosures}

Human subjects: Consent was obtained by all participants in this study. Conflicts of interest: In compliance with the ICMJE uniform disclosure form, all authors declare the following: Payment/services info: All authors have declared that no financial support was received from any organization for the submitted work. Financial relationships: All authors have declared that they have no financial relationships at present or within the previous three years with any organizations that might have an interest in the submitted work. Other relationships: All authors have declared that there are no other relationships or activities that could appear to have influenced the submitted work.

\section{References}

1. Fernandez-Bustamante A, Frendl G, Sprung J, et al.: Postoperative pulmonary complications, early mortality, and hospital stay following noncardiothoracic surgery: a multicenter study by the Perioperative Research Network Investigators. JAMA Surg. 2017, 152:157-66. 10.1001/jamasurg.2016.4065

2. Maw AM, Hassanin A, Ho PM, et al.: Diagnostic accuracy of point-of-care lung ultrasonography and chest radiography in adults with symptoms suggestive of acute decompensated heart failure: a systematic review and meta-analysis. JAMA Network Open. 2019, 2:e190703. 10.1001/jamanetworkopen.2019.0703

3. Lichtenstein DA, Mezière GA: Relevance of lung ultrasound in the diagnosis of acute respiratory failure*: the BLUE protocol. Chest. 2008, 134:117-125. https://doi.org/10.1378/chest.07-2800

4. Noble VE, Murray AF, Capp R, Sylvia-Reardon MH, Steele DJR, Liteplo A: Ultrasound assessment for extravascular lung water in patients undergoing hemodialysis: time course for resolution. Chest. 2009, 135:1433-39. https://doi.org/10.1378/chest.08-1811

5. Vitturi N, Dugo M, Soattin M, Simoni F, Maresca L, Zagatti R, Maresca MC: Lung ultrasound during hemodialysis: the role in the assessment of volume status. Nephrol. 2014, 46:169-74. https://doi.org/10.1007/s11255-013-0500-5

6. Vitturi N, Soattin M, Allemand E, Simoni F, Realdi G: Thoracic ultrasonography: A new method for the work-up of patients with dyspnea. J Ultrasound. 2011, 14:147-51. 10.1016/j.jus.2011.06.009 